\title{
Метод поиска выпуклых полиэдров с заданной точечной группой симметрии
}

\author{
Войтеховский Ю.Л. ${ }^{1,2}$, Степенщиков Д.Г. ${ }^{2}$ \\ ${ }^{1}$ Санкт-Петербургский горный университет, voytekhovskiy_yul@pers.spmi.ru \\ ${ }^{2}$ Геологический институт КНЦ РАН, Anamumbl, stepen@geoksc.apatity.ru
}

Аннотация. В предыдущих работах авторов перечислены и охарактеризованы точечными группами симметрии (т.г.с.) все выпуклые 4- .. 12-эдры и простые 13- .. 16-эдры (всего 27146775). Среди них найдены представители 24 кристаллографических $(1,-1,2, m, 3,222, m m 2,4,-4,2 / m, 32,-6,3 m, 4 m m, m m m,-42 m,-6 m 2$, $-3 m, 6 m m, 23,4 / m m m, 6 / m m m,-43 m, m-3 m)$ и 20 некристаллографических $(5 m, 7 m,-8 m 2,8 m m, 9 m,-10 m 2,-5 m$, $10 \mathrm{~mm}, 11 \mathrm{~m},-12 \mathrm{~m} 2,-7 \mathrm{~m},-14 \mathrm{~m} 2,8 / \mathrm{mmm},-18 \mathrm{~m} 2,10 / \mathrm{mmm},-22 \mathrm{~m} 2,12 / \mathrm{mmm},-26 \mathrm{~m} 2,14 / \mathrm{mmm},-3-5 m)$ т.г.с. Неизвестны представители 8 кристаллографических т.г.с.: 422, 4/m, -3, 6, 622, 6/m, m-3, 432. В этой статье дан метод поиска выпуклых полиэдров с заданными числом вершин (или граней) и т.г.с. Он основан на ограниченном переборе симметрических $(0,1)$-матриц специального вида, моделирующих матрицы смежности полиэдрических (3-связных планарных) графов.

Ключевые слова: выпуклый полиэдр, точечная группа симметрии, полиэдрический граф, матрица смежности

\section{A method to find convex polyhedra with a given symmetry point group}

\author{
Voytekhovsky Y.L. ${ }^{1,2}$, Stepenshchikov D.G. ${ }^{2}$ \\ ${ }^{1}$ Saint-Petersburg Mining University, voytekhovskiy_yul@pers.spmi.ru \\ ${ }^{2}$ Geological Institute of KSC RAS, Apatity, stepen@geoksc.apatity.ru
}

Abstract. All the convex 4- to 12-hedra and simple 13- to 16-hedra (27146775 in total) are enumerated and characterized by the symmetry point groups in the previous author's works. The representatives of 24 crystallographic $(1,-1,2, m, 3,222, m m 2,4,-4,2 / m, 32,-6,3 m, 4 m m, m m m,-42 m,-6 m 2,-3 m, 6 m m, 23,4 / m m m, 6 / m m m,-43 m, m-3 m)$ and 20 non-crystallographic $(5 m, 7 m,-8 m 2,8 m m, 9 m,-10 m 2,-5 m, 10 m m, 11 m,-12 m 2,-7 m,-14 m 2,8 / m m m,-18 m 2$, $10 / \mathrm{mmm},-22 \mathrm{~m} 2,12 / \mathrm{mmm},-26 \mathrm{~m} 2,14 / \mathrm{mmm},-3-5 \mathrm{~m})$ symmetry point groups are among them. The representatives of 8 crystallographic symmetry point groups are unknown: 422, 4/m, -3, 6, 622, 6/m, m-3, 432. A method to find convex polyhedra with a given number of vertices (or facets) and symmetry point group is given in the paper. It is based on the limited enumeration of the special symmetric $(0,1)$-matrices, which model the adjacency matrices of polyhedral (3-connected planar) graphs.

Key words: convex polyhedron, symmetry point group, polyhedral graph, adjacency matrix.

\section{Введение}

В предыдущих работах авторов (Войтеховский, Степенщиков, 2008 a, б) с помощью оригинальных алгоритмов и компьютерных программ впервые перечислены и охарактеризованы точечными группами симметрии (что адаптирует весьма рутинный результат к задачам кристалломорфологии) все выпуклые 4- .. 12-эдры и простые 13- .. 16-эдры (всего 27146775). Среди них есть представители 24 кристаллографических $(1,-1,2, m, 3,222, m m 2,4,-4,2 / m, 32,-6,3 m, 4 m m, m m m,-42 m$, $-6 m 2,-3 m, 6 m m, 23,4 / m m m, 6 / m m m,-43 m, m-3 m)$ и 20 некристаллографических $(5 m, 7 m,-8 m 2,8 m m$, $9 m,-10 m 2,-5 m, 10 m m, 11 m,-12 m 2,-7 m,-14 m 2,8 / m m m,-18 m 2,10 / m m m,-22 m 2,12 / m m m,-26 m 2$, $14 / \mathrm{mm},-3-5 m$ ) точечных групп симметрии (т.г.с.). Среди столь огромного многообразия выпуклых полиэдров не оказалось представителей 8 кристаллографических т.г.с.: 422, 4/m, -3, 6, 622, 6/m, m-3, 432. Очевиден вопрос: как систематически найти выпуклые полиэдры с указанными т.г.с.? Интерес состоит именно в систематическом поиске, начиная с простейших (с минимальным числом граней или вершин, что эквивалентно в силу дуального перехода) полиэдров, поскольку примеры выпуклых полиэдров с любыми т.г.с. даны, например, в (Попов, Шафрановский, 1964, с. 336-357). 


\section{Memod}

В статье (Voytekhovsky, 2016) предложен способ «именования» выпуклого полиэдра, позволяющий зафиксировать его комбинаторный тип (набор и способ соединения граней) в численном коде (имени). Последовательность операций следующая. (1) Нумеруем все вершины полиэдра числами от 1 до n. (2) Строим матрицу смежности $\mathrm{n} \times \mathrm{n}$ : на пересечении строки и столбца ставим 1 , если соответствующие вершины соединены ребром (смежны) и 0 - в противном случае. (3) В силу симметричности матрицы она фиксируется верхним треугольником, который выписываем построчно. Полученная последовательность единиц и нулей представляет собой бинарный код (легко преобразуемый в десятичный). По имени-коду рёберный граф полиэдра восстанавливается однозначно. На рисунке 1 показана последовательность операций для тетраэдра, его бинарный код 11111 , десятичный 63.

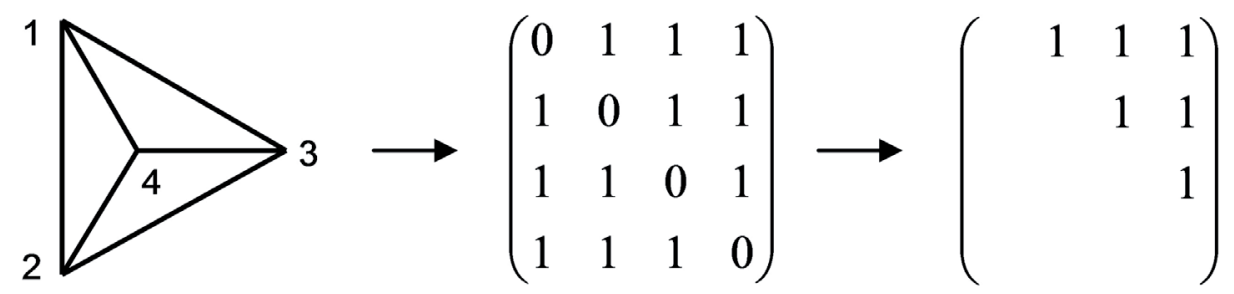

Рис. 1. Алгоритм нахождения имени (численного кода) выпуклого полиэдра через его рёберный граф и матрицу смежности вершин.

Fig. 1. Algorithm of finding the name (numerical code) of a convex polyhedron through its adjoint graph and adjacency matrix of vertices.

Легко показать, что для выпуклых полиэдров с числом вершин $\mathrm{n}>4$ при разной нумерации вершин будут получаться различные имена. Имеет место теорема: число имён выпуклого полиэдра равно n! / п.г.а., где п.г.а. - порядок группы автоморфизмов - численная характеристика т.г.с. Так, на рисунке 2 показаны все имена тетрагональной пирамиды (т.г.с. $4 m m$, п.г.а. 8 ): 5 ! / $8=15$.

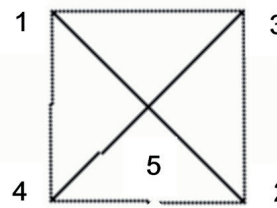

507

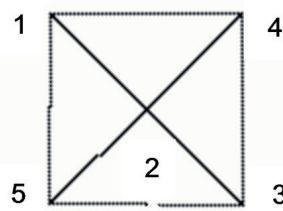

766

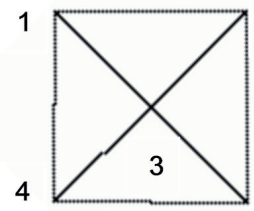

943

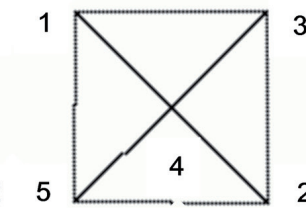

509

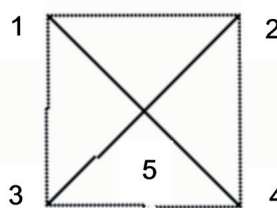

863

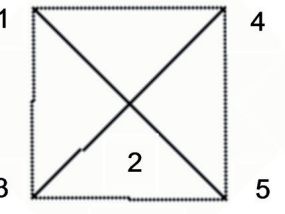

955

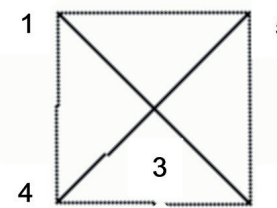

510

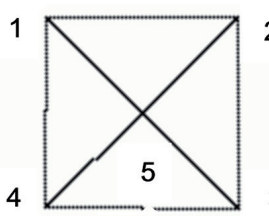

751

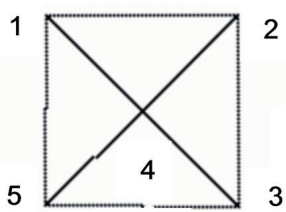

759

Рис. 2. Имена тетрагональной пирамиды, получаемые при различных нумерациях вершин.

Fig. 2. Names of a tetragonal pyramid obtained by different indexing of vertices. 
Из теоремы следует алгоритм поиска выпуклого полиэдра с заданной т.г.с. (1) Фиксируем класс n-вершинников, в котором будем искать выпуклый полиэдр с заданной т.г.с. и, следовательно, известным п.г.а. (2) Перечисляем бинарные имена-коды, соответствующие верхнему треугольнику матрицы смежности, с учётом теоремы, определяющей их точные нижнюю и верхнюю границы (Voytekhovsky, 2017). (3) Проверяем соответствующие рёберные графы на полиэдричность (3-связность и планарность). (4) Множество имён полиэдрических графов разбиваем на классы эквивалентности по признаку сводимости друг к другу симметричными перестановками строк и столбцов матриц смежности. Искомый полиэдр может находиться только в классе, содержащем n! / п.г.а. имён. (5) При наличии такового (таковых) убеждаемся в его (их) принадлежности к заданной т.г.с. Распознавание возможно по структуре группы перестановок строк и столбцов, сохраняющих матрицу смежности (Калужнин, Сущанский, 1979).

\section{Заключение}

Описанный алгоритм позволяет с ростом $\mathrm{n}$ рано или поздно найти выпуклый полиэдр с любой т.г.с. Единственный 4-вершинник - он же 4-гранник (тетраэдр) - имеет п.г.a. 24, т.г.с. $-43 m$. Два 5-вершинника попадают в разные классы: п.г.а. 8, т.г.с. $4 m m$ и п.г.а. 12, т.г.с. $-6 m 2$. Из семи 6-вершинников три различимы уже по п.г.а.: п.г.а. 48, т.г.с. $m-3 m$; п.г.а. 12 , т.г.с. $-6 m 2$; п.г.а. 10 , т.г.с. $5 m$. Два 6-вершинника различимы лишь по т.г.с.: п.г.а. 2, т.г.с. 2 и $m$. Наконец, ещё два 6-вершинника неразличимы и по т.г.с.: п.г.а. 4, т.г.с. $m m 2$. Таким образом, все стадии алгоритма нужны уже на ранней стадии поиска. Очередная задача - отыскание простейших выпуклых полиэдров с т.г.с. $422,4 / m,-3,6,622,6 / m, m-3,432$, начиная с непростых 13-вершинников.

\section{Литература}

1. Войтеховский Ю.Л., Степенщиков Д.Г. Комбинаторная кристалломорфология. Кн. IV. Выпуклые полиэдры. Т. І. 4- ... 12-эдры. Апатиты: Изд-во КНЦ РАН. 2008 а. 833 с.

2. Войтеховский Ю.Л., Степенщиков Д.Г. Комбинаторная кристалломорфология. Кн. IV. Выпуклые полиэдры. Т. ІІ. Простые 13- ... 16-эдры. Апатиты: Изд-во КНЦ РАН. 2008 б. 828 с.

3. Калужнин Л.А., Сущанский В.И. Преобразования и перестановки. М.: Наука. 1979. 112 с.

4. Попов Г.М., Шафрановский И.И. Кристаллография. М.: Высшая школа. 1964. 370 с.

5. Voytekhovsky Y.L. How to name and order convex polyhedra // Acta Cryst. 2016. A72. P. 582-585.

6. Voytekhovsky Y.L. Convex polyhedra with minimum and maximum names // Acta Cryst. 2017. A73. P. 271-273. 